\title{
Uplink Performance Evaluation of CDMA Communication System with RAKE Receiver and Multiple Access Interference Cancellation
}

\author{
Ayodeji J. Bamisaye, Michael O. Kolawole \\ Department of Electrical and Electronics Engineering the Federal University of Technology, Akure, Nigeria \\ E-mail:ayobamisaye@yahoo.com and kolawolm@yahoo.com \\ Received January 26, 2010; revised March 2, 2010; accepted April 10, 2010
}

\begin{abstract}
In CDMA communication systems, all the subscribers share the common channel. The limitation factor on the system's capacity is not the bandwidth, but multiuser interference and the near far problem. This paper models CDMA system from the perspective of mobile radio channels corrupted by additive white noise generated by multipath and multiple access interferences. The system's receiver is assisted using different combining diversity techniques. Performance analysis of the system with these detection techniques is presented. The paper demonstrates that combining diversity techniques in the system's receivers markedly improve the performance of CDMA systems.
\end{abstract}

Keywords: CDMA, Multipath Diversity, Multiple Access Interference Cancellation, Rake Receiver, Parallel Interference Cancellation

\section{Introduction}

The mobile radio channel plays fundamental limitations on the performance of wireless communication systems. The transmission path between the transmitter and the receiver can vary from simple line-of-sight to one that is severely obstructed by buildings, mountains, and foliage. Unlike wired channels that are stationary and predictable, radio channels are extremely random and do not offer easy analysis. Even the speed of motion impacts how rapidly the signal level fades as mobile terminal moves in space. Modelling the radio channel has historically been one of the most difficult parts of mobile radio system design, and is typically done in a statistical fashion, based on measurements made specifically for intended communication system or spectrum allocation. Code Division Multiple Access (CDMA) is a multiple access technology that utilizes direct-sequence spread spectrum (DS-SS) techniques. With this technology comes a paradigm shift, including the use orthogonal or nearly orthogonal codes (so-called spreading sequences) to modulate the transmitted bits. Contrary to the conventional frequency division multiple access (FDMA) and time division multiple access (TDMA) systems where noise rejection deals, primarily with out-of-band noise, a CDMA system concerns mostly with inband noise. This noise may come from self-jamming (or self-noise), background noise, man-made noise, inter-modulation, or noise generated in the receiver [1,2]. If one can reduce the unwanted in-band noise, such reduction translates directly into improved performance. Undesired noise comes from many different sources. This noise may come from natural or human sources. Naturally occurring noise includes atmospheric disturbances, background noise, and thermal noise generated in the receiver itself. Through careful engineering, the effects of many unwanted signals can be reduced [3]. DS-CDMA is a multiple access system, where multiple users share a limited resource, the frequency. Conventional asynchronous DS-CDMA systems allow each user to transmit and receive independently. Each receiver performs a simple correlation between the received baseband signal and the corresponding user's spreading sequence. In a low-noise channel with orthogonal spreading sequence,this approach would be optimal. Due to the synchronicity of users and the need to support numerous users, such orthogonality is impossible, even on a hypothetical AWGN (additive white Gaussian noise) channel [4]. Thus, system performance rendered multiple-access interference (MAI) limited, and channel utilization is correspondingly low.

This paper models CDMA system from the perspective 
of mobile radio channels corrupted by additive white noise generated by multipath and multiple access interferences. The system's receiver is assisted using different combining diversity techniques. Performance analysis of the model with different detection techniques will be presented.

\subsection{Mobile Radio Channel Model}

Mobile radio channel is one of the most important elements in the mobile communication systems. When the signals are transmitted through Mobile radio channel, it is affected by shadow or large scale fading. Mobile communication is affected by multipath fading in addition to shadow fading. Multipath fading is caused by atmospheric, scattering, and reflection from building and other object. Multipath channel can be classified as discrete (consisting of resolvable multipath components) and diffuse (consisting of irresolvable multipath components) $[5,6]$. Consequently, the multipath fading could affect the transmitted signals in two ways: due to dispersion (also called time spreading or frequency selectivity), and due to time variant behaviour of the channel (due to motion of the receiver or changing environment such as of foliage or movement of reflectors and scatter). This means that the impulse response $h(t, \tau)$ of mobile radio channel is time variant [6,7], and if $h(t, \tau)$ has a zero mean, then the envelope $|h(t, \tau)|$ has a Rayleigh distribution with its probability density function described by

$$
p(r)=\frac{r}{\sigma^{2}} \exp \left[-\frac{r^{2}}{2 \sigma^{2}}\right]
$$

where $r$ is time dependent received signal (i.e., $r(t)=|r| e^{-j \phi(t)}, \phi$ is its arbitrary phase) whose line of sight (LOS), specular, and diffuse components are assumed bounded in narrowband form, and $\sigma^{2}$ is the total power in the multipath signal. In this case the total power is considered to have zero-mean, amplitude fading. Otherwise, if the impulse response has a non-zero mean, then the envelope mobile radio channel would have a Rician distribution with its probability density function expressed as:

$$
p(r)=\frac{r}{\sigma^{2}} \exp \left[\frac{-\left(r^{2}+s^{2}\right)}{2 \sigma^{2}}\right] I_{o}\left(\frac{r^{2}}{\sigma^{2}}\right)
$$

where $s^{2}$ is the power of the line-of-sight component, and $I_{o}$ denotes the zeroth order Bessel function of the first kind argument (.). The Rayleigh distribution is a special case of Rician distribution when $s=0$; i.e., where the LOS component is negligible.

In most general case, the channel can be modelled as linear time-variant system [2] giving the response of the channel at time $(t)$ to an impulse at time $(t-\tau)$. If $x(t)$ represents the transmitted (or emitted) signal through noiseless mobile radio channels, the received signal, $r(t)$ can be expressed as

$$
r(t)=\int_{-\infty}^{\infty} h(t, \tau) x(t-\tau) d \tau
$$

If the channel information is considered, its distributed channel impulse response may be expressed as

$$
h(t, \tau)=\sum_{j=0}^{N-1} \beta_{j}(t) \delta\left(\tau-\tau_{j}\right)
$$

In view of (4) in (3), we write modified channel received signal:

$$
r(t)=\sum_{j=0}^{N-1} \beta_{j}\left(\tau_{j}\right) x\left(t-\tau_{j}\right)
$$

$\beta_{j}(t)$ is complex amplitude, $\tau_{j}$ is the path delay, and $N$ is the number of multipath components. In the wideband channel where the delay-line model had a large number of taps, not all the multipath components are likely to fade simultaneously. This may be used as a multipath diversity to improve the received signal SINR (carrier to interference plus noise ratio). Rake receiver, for instance, can be used to mitigate the effect of fading if the transmitted signal bandwidth is larger than the coherence bandwidth; a practical example is in the widebandCDMA (WCDMA).

\subsection{Rake Receiver}

Conventional matched filters are single path detectors. In practice, when the transmitted signal passes through mobile radio channel, duplicates of the transmitted signal are generated by reflection, refraction, and diffraction, and the signal power is distributed in multipath. In CDMA system, the transmitted signal bandwidth is much larger than the coherent bandwidth of the channel, in which case the channel is frequency selective [4,8,9]. For the frequency-selective channels, the received signals are multiple copies of the transmitted signals with different channel delays and fading, combining the multipath components as multipath diversity. Thus, if one of the multipath components is attenuated by fading, some others may not be and the receiver could use unfaded components to make the decision. The idea behind the rake reception technique is that the signals propagating through different multipath are received in individual fingers of the rake receiver and the outputs from these fingers are then coherently combined to provide the input signal for the symbol decision. The received signal is chip matched and sampled at the chip rate. Figure 1, illustrates the structure of a typical rake receiver in which $s_{k}^{*}(i)$ and $c_{k, m}^{*}(i)(k=1,2, \ldots, K ; m=1,2, \ldots, M)$ represent, respectively, the complex conjugate of chipchannel matched sampled signature sequence of the user 


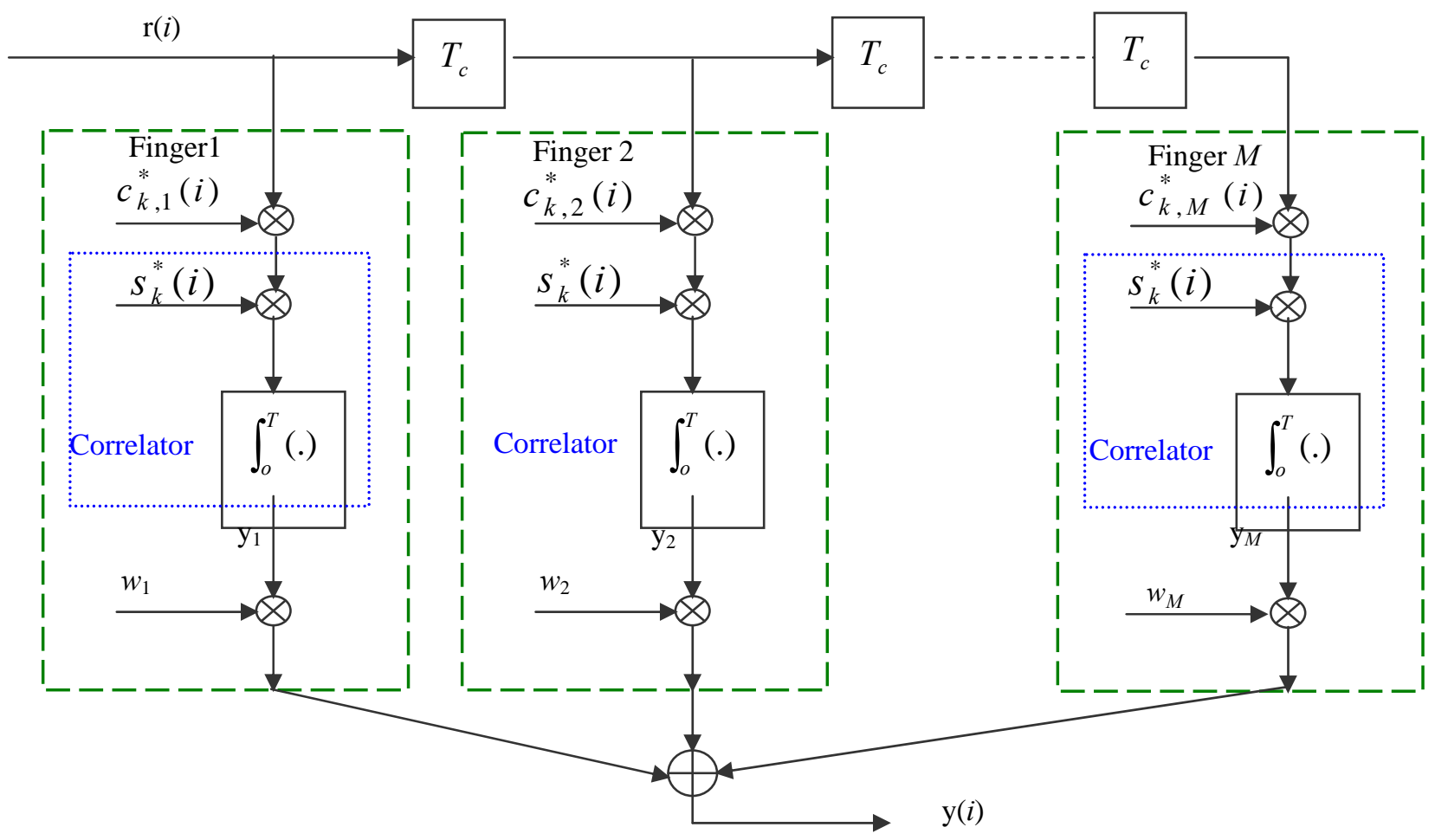

Figure 1. Rake Receiver with $M$ fingers.

of interest and the complex conjugate estimate of the impulse response.

Then, the decision on the transmitted information bit is based on the sum of the individual correlator's outputs. In our study, the dimension of each correlator equals the system processing gain, and then the output of each finger $y(i)$ is given after channel is matched and correlating:

$$
\begin{aligned}
y(i)= & \sum_{j=1}^{P G} \sum_{m=1}^{M} \sum_{k=1}^{K} c_{k, m}^{*}(i) s_{k, j}^{*}(i) r_{k}(i) \text { for } w_{m}=1 \\
& \text { (where } m=1,2, \ldots, M)
\end{aligned}
$$

where $P G$ is the processing gain, $c_{k, m}^{*}(i)$ is the complex conjugate estimate of the channel's impulse response, $s_{k, j}^{*}(i)$ is the complex conjugate of chip-matched sampled signature sequence of the users of interest.

\subsubsection{Multistage Receivers}

The input signal of a multiuser detector is the output of the matched filter bank, or rake receiver. Almost all modern multiuser detection techniques deal with the output of the matched filters bank and the cross-correlation information of all users in the system. Expressions (1) through (6) assumed noiseless cases, which is not valid in real life situation. If the time-dependent interference components, $n(t)$, are assumed additive, the output of the conventional matched filters receiver for single user $k$ can be expressed as:

$$
y_{k}(i)=\sum_{i=1}^{P G} c_{k}^{*}(i) s_{k}^{*}(i) r_{k}(i)+\sum_{i=1}^{P G} c_{k}^{*}(i) s_{k}^{*}(i) n(t)
$$

In the case of multiple $\mathrm{K}$ active users, the received signal in the receiver is

$$
r(t)=\sum_{k=1}^{K} r_{k}(t)+n(t)
$$

So, the modified output of the conventional matched filter receiver $\mathrm{y}_{k}$ for the $k$ th user is

$$
\begin{aligned}
& y_{k}=\sum_{i=1}^{P G} r_{k}(i) c_{k}^{*}(i) s_{k}^{*}(i) \\
& +\sum_{\substack{j=1 \\
j \neq k}}^{K} \sum_{i=1}^{P G} r_{j}(i) c_{k}^{*}(i) s_{k}^{*}(i)+\sum_{i=1}^{P G} c_{k}^{*}(i) s_{k}^{*}(i) n(t)
\end{aligned}
$$

Expression (9) consists of three terms. The first term is the desired information which gives the signal of the information bit (which is exactly what is sought). The second term is the result of multiple access interference (MAI), and the last term is due to noise. The second term typically dominates the noise so that one would like to remove its influence. Its influence is felt through the cross-correlation between the chip sequences and the device-powers of users. If one knew the cross-correlations and the powers, then one would attempt to cancel the 
effect of one user upon another. This is, in fact, the intuitive motivation for interference cancellation scheme.

The difference between multistage receivers and successsive interference canceller (SIC) receivers is that instead of using previous bit decisions to cancel interfereence from desired user's signal as in SIC, tentative decisions on each user are used to improve signal quality [10]. Receiver structure is called multistage, since when decisions are made, they can be used to either make a final decision on data or to enhance the signal through cancellation. Reference signals are based on initial bit estimates, which are then subtracted from received signal to produce a cleaned spread signal for next stage. Since all signals are detected at each stage simultaneously, multistage receivers are also called parallel interference cancellers (PIC). A two-user multistage receiver is shown in Figure 2. The p-stage receiver outputs are $y_{k}^{p-1, p}(t)$, where $p=1,2, \ldots$.

Instead of a conventional (matched filter) receiver front end, a SIC receiver (or a decorrelator) can be used [11]. Performance of PIC is best when received signal powers are equal. Capacity of the system is limited by hardware.

\subsection{Simulation and Results}

Performance evaluations were performed for different scenarios using:

1) Conventional matched filters receiver with PIC (parallel interference cancellation);

2) Rake receiver with PIC;

3) Rake receiver with Decorrelating Detector and MMSE; and

4) Conventional matched filters receiver and rake receiver.

For each analysis, the number of users' symbols transmitted is clearly stated. It is assumed that the system uses unencoded BPSK signal, as well as no pulseshaping filter. Each transmitted MS within each loop sends a block of data bits of known length. The input of MUD (multi user detection) is the soft decision of either rake receiver or conventional matched filters. In the simulation, the sub-optimum linear MUD decorrelating detector, linear minimum mean squared error (LMMSE) detector, and nonlinear sub-optimum PIC were built for the multishot model.

In relation to the decision operation, the hard decision output of received data is obtained by passing the soft decision output through the design circuit, which represents any sign function for BPSK. Also, we make the number of channel paths $P$ equal the number of fingers $M$ to make the rake receiver simpler in structure. In a less equivalent case, that is, when $\mathrm{M}<\mathrm{P}$ or $\mathrm{M}>\mathrm{P}$, the system performance degrades. Therefore, the matched case provides the optimally achievable performance reference.

\subsubsection{Performance of Conventional Matched Filters Receiver with PIC}

Simulations were performed for 10 active users sending 10 symbols within each loop. The maximum loop equals to 100 . The users send a total of 10000 symbols. The data are then spread by Walsh code and scrambled by Gold code with processing gain of 32. The channel parameters are updated randomly in each loop.

Simulation results are shown in Figure 3, which demonstrates progressive improvement in the system performance with diversity combining techniques in cancelling multiple access interferences.

\subsubsection{Performance of Conventional Matched Filters Receiver and Rake Receiver}

Similar parameters used in 1.3.1 are used in this analysis except the processing gain, PG, which is increased to 64 . In addition, the channel parameters are updated randomly in each loop and the number of paths for each user changes randomly over (2-6). Two tests were carried out in this subsection: (i) to investigate the effect of rake receiver in the system for a single user and (ii) to investigate the effect of rake receiver and conventional (matched filter) receiver with a range of channel paths.

Figure 4 shows the performance with rake receiver and without rake receiver for a single user. In the receiver, 'equal gain combining' method is used in rake. The analysis demonstrates that lowering the SNR does not improve the system when SNR equals $20 \mathrm{~dB}$, the BER is $10-2.9$ and $10-1.9$ respectively for conventional matched filter receiver and rake receiver. In contrast, improvement in system performance can be achieved by increasing SNR in both schemes.

In the case of test (ii), an AGWN is assumed in the channel paths. The number of users and the processing gain are decreased to 6 and 8 respectively, while other parameters are the same as previous simulations. The channel parameters are updated randomly in each loop, and the simulation computes for the different values of channel path, as shown in Figure 5.

Figure 5 shows the performance of the rake and conventional receivers for a number of multipath scenarios. In the case of one path, the system performance is the same for matched filter (MF) and rake receiver. But in the case of multipaths; for example, (4-path) the system performance with conventional matched filter degrades, whereas the system performance with rake receiver improves. Also, for the case of (7-path) the performance of MF becomes very poor, but improves markedly with rake receiver. As a result, the system performance can be deduced as increasing when diversity scheme is employed. Under Gaussian noise as interferer, the system performance improves with in creasing channel path with the rake receiver, which might not be optimal in real life situation. 


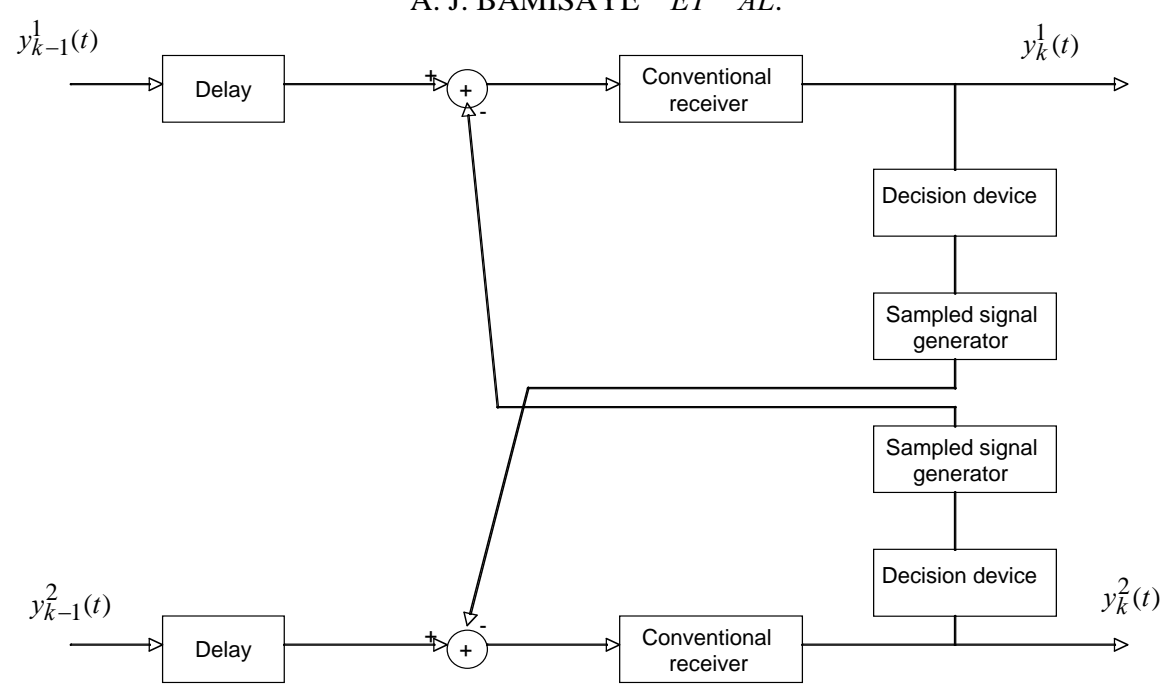

Figure 2. A two-user multistage receiver.

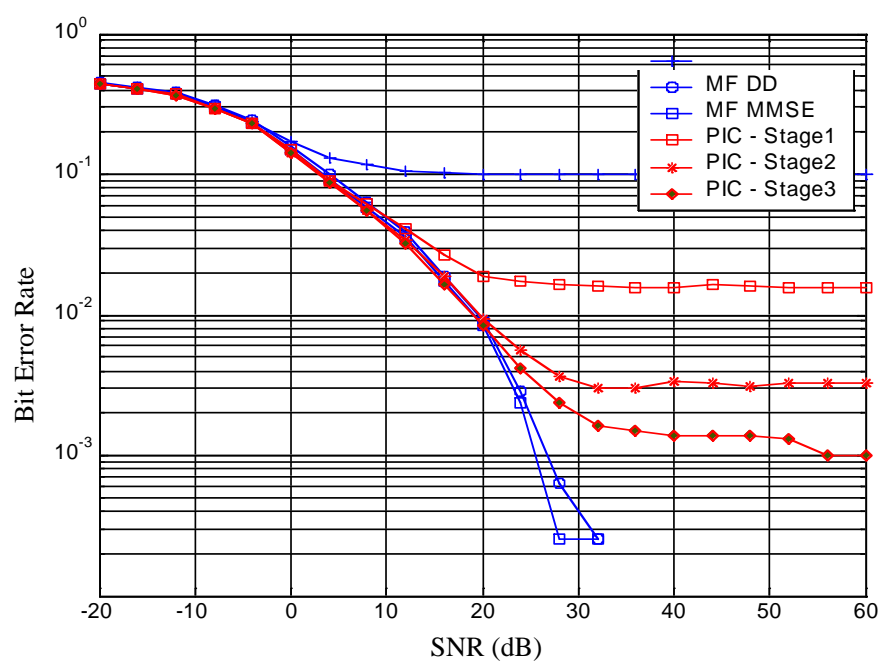

Figure 3. CDMA system performance evaluation using conventional matched filter (MF), with different diversity techniques: decorrelating detector (DD), minimum mean squared error (MMSE), and three-stage parallel interference cancellation (PIC).

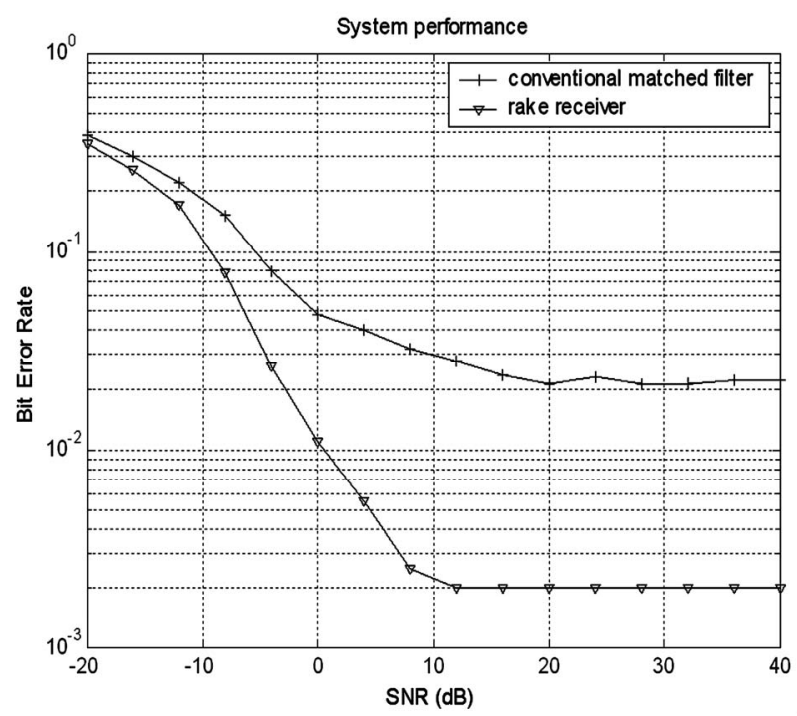

Figure 4. System performance of Rake receiver and conventional matched filters' receiver. 


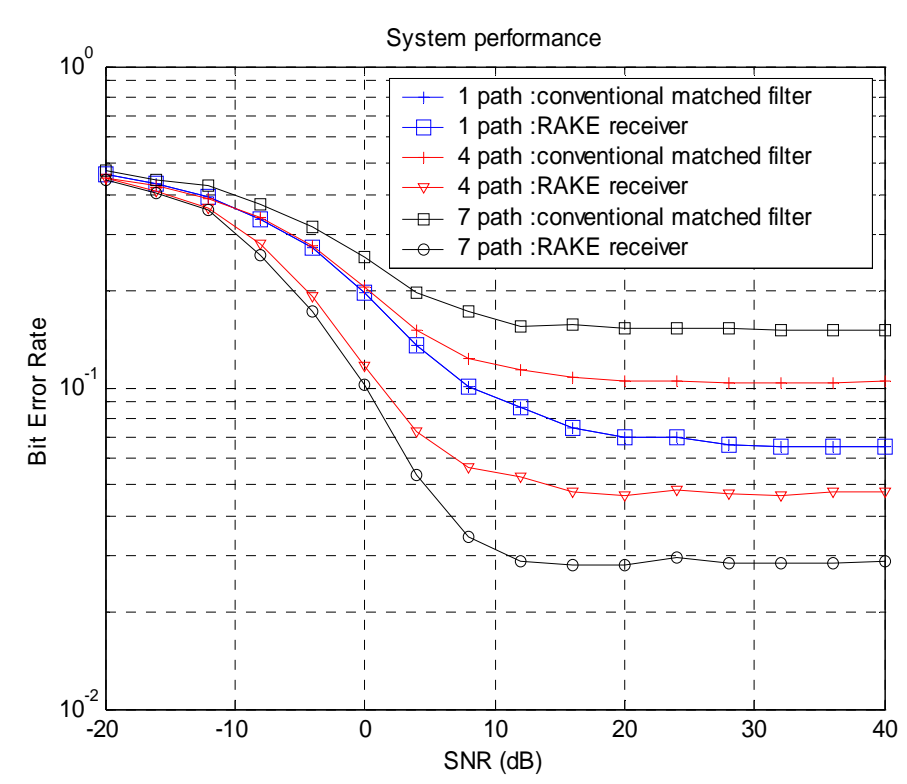

Figure 5. System performance of Rake receiver and conventional matched filters' receiver with different number of channel paths.

\subsubsection{Performance of RAKE Receiver with Decorrelating Detector and MMSE}

Similar parameters used in 1.3.1 are used in this analysis. In addition, the channel parameters are updated randomly in each loop, and the number of paths for each user changes randomly between 2 and 5. Figure 6 clearly shows the importance of multiuser detection (MUD). The figure shows that for one path, marginal improve- ment in system performance is gained whether conventional matched filters receivers are used or in combination with decorrelating detector (DD) or with minimum mean squared error (MMSE) schemes. Note that the correlation matrix only contains the information of multiple access interferences in the first path and treated the other paths as AWGN. On the other hand, system performance improves markedly using rake receiver and MUD when taking the multipath into account; a finding consistent with that of [11].

\subsubsection{Performance of RAKE Receiver with Parallel Interference Cancellation}

For this simulation, 10 active users send 10 symbols respectively within each loop. The maximum loop is equal to 150 , with a total of 15000 symbols sent. The data are then spread by Walsh code and scrambled by Gold code with performance gains, PGs, of 16 and 64. The channel parameters are updated randomly in each loop, and the number of channel path change randomly between 2 and 4. The received signal is processed with rake and conventional matched filter respectively. Three-stage parallel interference cancellation is employed to cancel the multiple access interference. As shown in Figure 7, the performance improved by using rake receiver instead of conventional matched filters.

\subsubsection{Performance with Variable Processing Gain} In this simulation, 8 active users send 10 symbols respectively within each loop. The maximum loop is equal to 100 , totalling 8000 symbols sent by the users. The data are then spread by Walsh code and scrambled by Gold code with performance gains of 16 and 64. The channel parameters are updated randomly in each loop, and the number of channel path change randomly between 2 and 4 . The received signal is processed with rake and conventional matched filters respectively. Decorrelating detector is employed to cancel the multiple access interference. Figure 8 indicates the processing gain influence to the system performance. The results demonstrate that large processing gains translate to high performance gain of the system.

\subsection{Conclusions}

This paper has modelled a CDMA system from the perspective of mobile radio channels corrupted by additive white noise generated by multipath and multiple access interferences. The system's receiver was assisted with different combining diversity schemes. The simulation is focused on the most important factors that will influence the performance of the CDMA systems using multi user detection method and interference cancellations scheme. Performance analysis of the system using the different detection techniques was presented. The paper estabLished that diversity-combining techniques markedly im- 


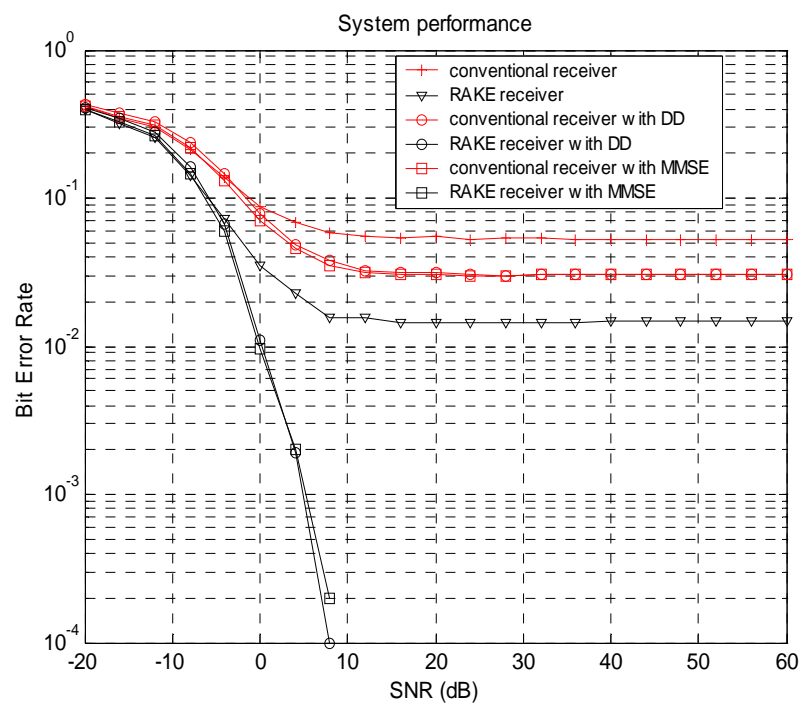

Figure 6. System performance of Rake receiver with decorrelating detector (DD) and minimum mean squared error (MMSE).

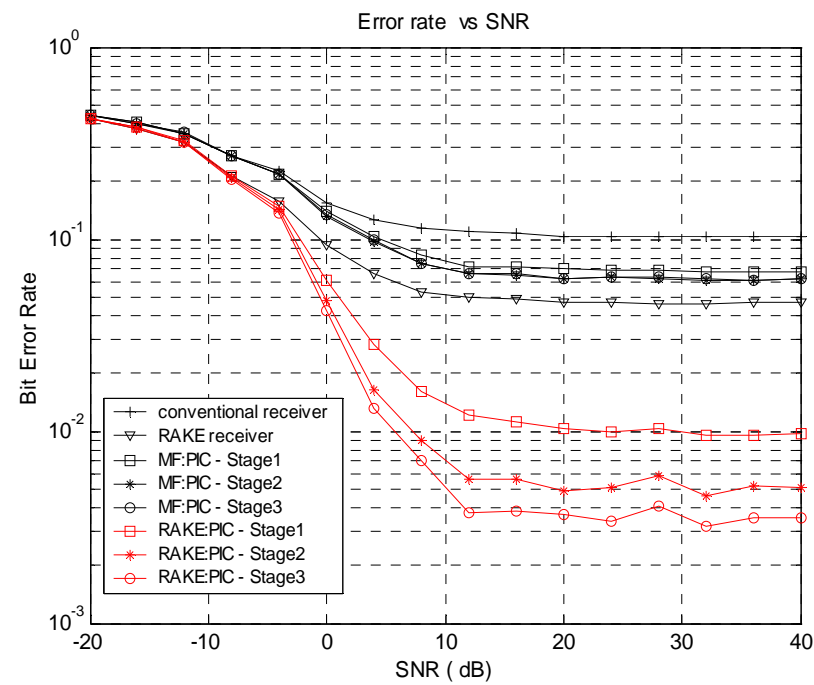

Figure 7. The system performance of RAKE receiver with three-stage parallel interference cancellation (PIC).

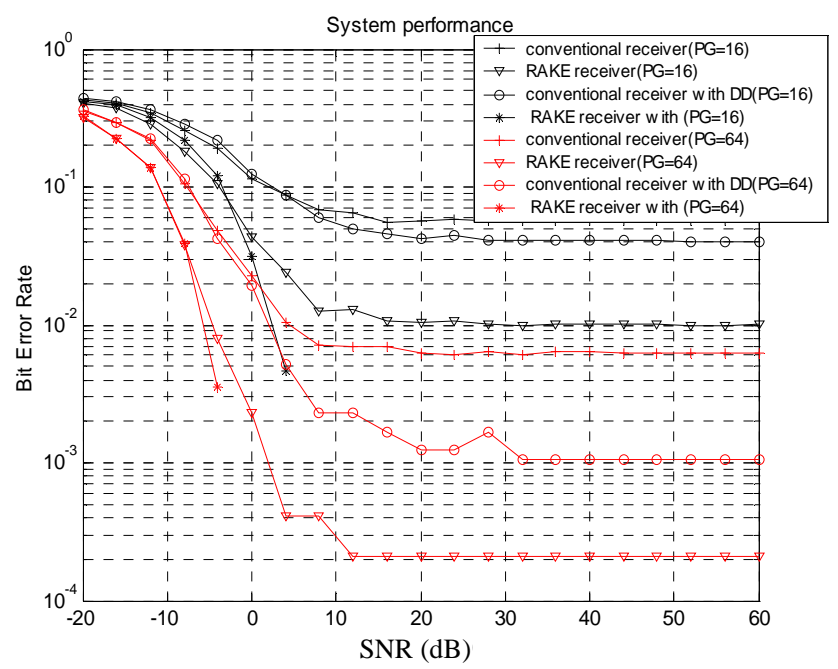

Figure 8. System performance of RAKE receiver with different. 
prove the performance of CDMA systems. Also, an important factor in reducing the multiple access interfereence, is the processing gain which the model employed.

\section{References}

[1] D. Torrieri, "Principle of Spread-Spectrum Communication Systems," Springer Science + Business Media, Boston, 2005.

[2] R. Prasad, "Universal Wireless Personal Communications,” Artech House, 2000.

[3] S. C. Yang, "CDMA RF System Engineering," British Library Cataloguing in Publication Data, II. Series, TK5103.2.Y36, Irvine, California, 1998.

[4] A. J. Bamisaye, "Effect of Multiuser Interference on Subscriber Location in CDMA Network,” M Eng Thesis, Department of Electrical and Electronics Engineering, the Federal University of Technology, Akure, 2009.

[5] A. J. Bamisaye and M. O. Kolawole, "Evaluation of Downlink Performance of a Multiple-Cell, Rake Receiver Assisted CDMA Mobile System," Wireless Sensor Network Journals, Vol. 2, No. 1, 2010, pp. 1-6.

[6] T. S. Rappaport, "Wireless Communications Principles \&
Practice,” IEEE Press, New York, Prentice-Hall, 2002.

[7] M. Frikel, B. Targui, F. Hamon and M. M’Saad, “Adaptive Equalization Using Controlled Equal Gain Combining for Uplink/Downlink MC-CDMA Systems,” International Journal of Signal Processing, Vol. 4, No. 3, 2008, pp. 230-237.

[8] A. Kansal, S. N. Batalama and D. A. Pados, “Adaptive Maximum SINR RAKE Filtering for DS-CDMA MultiPath Fading Channels," IEEE Journal on Selected Area in Communication, Vol. 16, No. 9, 1998, pp. 1765-1773.

[9] T. Kim, K. Ko, Y. Kim and D. Hong, "Performance Evaluation of Uplink MC-CDMA Systems with Residual Frequency Offset," IEICE Transactions on Communications, Vol. E89-B, No. 4, 2006, pp. 1455-1458.

[10] K. Vardhe, D. Reynolds and M. C. Valenti, “The Performance of Multi-User Cooperative Diversity in an Asynchronous CDMA Uplink," IEEE Transactions on Wireless Communications, Vol. 7, No. 2, 2008, pp. 19301940.

[11] X. B. Yu, X. D. Zhang, D. Z. Xu and G. G. Bi, "Uplink Performance of CWP-MC-CDMA with Space Diversity Combining Technique over Rayleigh Fading Channel," Journal of Electronics, Vol. 23, No. 6, 2006, pp. 837-841. 